\title{
Norois
}

Environnement, aménagement, société

\section{Vers un urbanisme affectif. Pour une prise en compte de la dimension sensible en aménagement et en urbanisme}

Toward an Affective Urbanism. Taking into Account Sensibility in Urban Planning

\section{Benoît Feildel}

\section{(2) OpenEdition \\ Journals}

Édition électronique

URL : http://journals.openedition.org/norois/4674

DOI : $10.4000 /$ norois. 4674

ISBN : 978-2-7535-2916-8

ISSN : $1760-8546$

\section{Éditeur}

Presses universitaires de Rennes

\section{Édition imprimée}

Date de publication : 30 juin 2013

Pagination : 55-68

ISBN : 978-2-7535-2882-6

ISSN : 0029-182X

\section{Référence électronique}

Benoît Feildel, « Vers un urbanisme affectif. Pour une prise en compte de la dimension sensible en aménagement et en urbanisme », Norois [En ligne], 227 | 2013, mis en ligne le 30 juin 2015, consulté le 01 mai 2019. URL : http://journals.openedition.org/norois/4674 ; DOI : 10.4000/norois.4674 


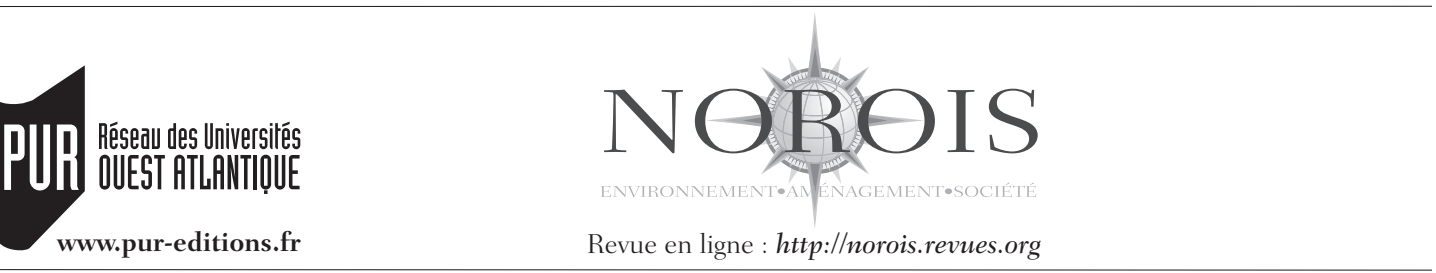

\title{
Vers un urbanisme affectif Pour une prise en compte de la dimension sensible en aménagement et en urbanisme
}

\author{
Towards an Affective Urbanism. Taking into Account Sensibility in Urban Planning
}

\author{
Benoît FEILDEL
}

\begin{abstract}
Maître de conférences en Aménagement de l'espace et urbanisme, École Polytechnique de l’Université de Tours, Département Aménagement, UMR 7324 CITERES, équipe Ingénierie du Projet d'Aménagement Paysage et Environnement, 33 allée Ferdinand-de-Lesseps - 37204 Touns cedex 03 (benoit.feildel@univ-tours.fr)
\end{abstract}

Résumé : On constate aujourd'hui un engouement croissant, dans le domaine de l'aménagement et de l'urbanisme, et plus généralement encore dans les sciences de l'espace, pour la thématique de l'affectivité. De plus en plus nombreux sont les auteurs qui soulignent l'importance et l'intérêt de prendre en compte cette donnée sensible. Souscrivant à cet objectif, l'article propose de montrer dans quelle mesure la dimension affective de la relation de l'être humain à son environnement constitue une connaissance utile à l'aménagement des espaces. Ainsi, nous soutenons que la relation affective à l'espace, incluant la façon de sentir, et surtout, la façon de se représenter la manière dont l'on sent, autrement dit de ressentir, est une dimension conséquente de l'organisation des espaces et qu'elle participe de l'agencement des spatialités individuelles et collectives. Enfin, en proposant une méthode pour appréhender le rapport affectif à l'espace, et sa construction à l'échelle biographique, nous suggérons une voie originale pour accéder à un vécu d'ordinaire difficilement préhensible.

Abstract: We notice nowadays a strong enthusiasm for affectivity in urban planning, and more generally in sciences concerned with the spatial conditions of society. More and more authors emphasize the importance and the need of taking into account sensitive data. Endorsing this goal, the purpose of the article is to show how the affective bond between man and environment provide useful knowledge for planning theory and practice. Thus we promote that affective bond to space, including the way we feel and the way we represent our feeling, is a subsequent dimension of place settlement, participating to individual and collective spatial organization. Finally, by proposing a method for measuring affective bond, and its biographical construction, we suggest a new way for approaching an experience that is usually quite difficult to reach.

Mots clés : rapport affectif - affectivité - sentir - ressentir - urbanisme - aménagement

Keywords: affective bond - affectivity - feeling - planning theory and practice 


\section{INTRODUCTION}

Le point de départ de notre réflexion peut être ainsi formulé : la dimension affective de la relation de l'être à son environnement, son « rapport affectif à l'espace »(Feildel, 2010), depuis les mécanismes qui président à sa construction, jusqu'aux conséquences pratiques et spatiales de ce lien, constitue une connaissance utile à l'aménagement des espaces. En effet, un nombre croissant d'auteurs (Manola, 2012; Audas, 2011 ; Feildel, 2004, 2010; Anderson et Holden, 2008; Damery, 2008; Labussière, 2007 ; Bigando, 2006; Thrift, 2004 ; Martouzet, 2002, 2007; Bochet et Racine, 2002) souligne l'intérêt de surmonter l'apparente difficulté que poserait la prise en compte de l'affectivité et propose ainsi de renouveler les conceptions qui relèvent à la fois de la description et de la compréhension des phénomènes spatiaux, mais aussi de l'organisation et de la transformation des espaces. Le présent article vise à montrer comment la dimension affective de la relation à l'espace - principalement documentée jusqu'à présent par les géographes, les sociologues et les psychosociologues - est susceptible d'intervenir à la fois dans la construction des représentations, des décisions et in fine des actions, qui vont participer aussi bien des logiques géographiques que des logiques projectives au fondement de l'agencement spatial des sociétés. À travers cette contribution, à la fois théorique et méthodologique, nous soutenons que la dimension affective de la relation entre l'être et ses différents espaces de vie est susceptible d'être comprise - et étudiée en tant que telle - comme dimension intervenant de multiples façons dans la pratique et l'organisation - intentionnelle ou non des lieux géographiques.

Pour ce faire, un préalable nous semble toutefois nécessaire qui nous invite à revenir sur le qualificatif «affectif » afin d'en préciser la portée et la place, entre les domaines du sentir et du ressentir. Car en cloisonnant sensations et affections, et en conférant une primauté - ne serait-ce que d'ordre temporel - aux premières sur les deuxièmes, les théories psychologiques opèrent une scission et un renversement qui sont préjudiciables à la compréhension de ces phénomènes en tant que tels, mais également à l'appréhension des phénomènes affectifs qui lient entre eux, les individus, les sociétés et leurs espaces. À la suite des pragmatistes, Dewey
([1915] 2010) et James ([1884] 2006) notamment, nous proposons de retravailler l'appréhension de l'ordre réel des choses et de questionner le primat donné à la sensation, à la perception sensible, sur lequel traditionnellement l'on s'accorde avant de considérer que celle-ci ne s'incarne dans un ressentir. Reconnaître, à la suite de Dewey ([1915] 2010), que «la perception émerge quand le souci envers les objets et leurs qualités porte à la conscience les exigences organiques d'attachement ", c'est placer de fait le sentir et le ressentir sur un même plan et, pour cela, envisager la relation d'étroite dépendance qu'ils entretiennent. Ainsi, nous revenons sur l'expérience sensible de ce qui apparaît, dans un premier temps, comme un donné, pour insister sur la manière - nécessairement située, temporellement, spatialement et socialement - d'éprouver la dimension spatiale de l'existence. Autrement dit, nous interrogeons la façon de ressentir (« re-sentir »), c'est-à-dire de conférer un sens particulier au vécu affectif.

Considérant que l'individu et le social sont engagés réciproquement dans la production des manières d'être et de sentir - ces manières, comme les qualifiait Durkheim, qui sont extérieures à l'individu et, à ce titre, qui sont douées d'un pouvoir de coercition en vertu duquel ils s'imposent à lui (Durkheim, 1895) - nous suggérons que la forme particulière que revêt pour un individu ou un groupe d'individus le vécu d'un état sensible, l'expérience des sensations de plaisir ou de déplaisir, a une influence sur l'organisation des espaces. En outre, nous faisons l'hypothèse que cette manière d'être sensible s'affirme aujourd'hui comme mode à part entière du «faire avec l'espace » (Lussault et Stock, 2010), comme un mode individuellement et collectivement déterminant dans les arrangements spatiaux de tous ordres, et soutenons réciproquement que les organisations spatiales, qui sont le reflet de nos sociétés, exercent elles aussi, en retour, une influence sur le sens que chacun confère à ses propres sensations et à celles des autres.

Dans un premier temps, nous nous intéressons aux conditions récentes de la reconnaissance en aménagement et en urbanisme du rapport affectif à l'espace. Puis, dans un deuxième temps, nous soulignons cette connivence entre senti et ressenti, et tâchons ainsi de rétablir l'association intime entre sensation et affection, tout en précisant la distinc- 
tion méthodologique entre ces deux termes. Enfin, dans un troisième temps, nous proposons une méthode pour atteindre une dimension de la réalité, celle des affections, que l'on considère comme difficilement préhensible, et montrons ainsi la portée et l'intérêt de ces questions pour l'aménagement et l'urbanisme.

\section{ESPACES ET PROJETS À L'ÉPREUVE DES AFFECTS}

On constate aujourd'hui un engouement croissant pour la thématique de l'affectivité. Pourtant, les affects ont longtemps été considérés comme une perfectibilité de l'âme humaine, dont on ne retenait au final que le caractère déstabilisateur. Cette opposition caricaturale par bien des aspects a vécu et, sous l'impulsion de disciplines aussi diverses que la neurologie, l'histoire, l'économie ou la sociologie, l'on redécouvre aujourd'hui les fonctions individuelles et sociales des affects (Elster, 2003). Par ailleurs, la revalorisation des affects dans nos sociétés est telle que certains auteurs pointent plus généralement un processus d'« émotionnalisation de la réflexivité » (Holmes, 2010), soulignant comment l'expérience affective est devenue, en l'espace de quelques décennies seulement, à la fois un mètre étalon de l'évaluation de l'accomplissement personnel et de la mesure d'une vie « réussie ».

Ce revirement, que l'on peut notamment mesurer à travers la montée en puissance de valeurs aussi diverses que l'hédonisme, l'empathie, la demande de reconnaissance, la sensibilité, s'exprime également dans le champ de l'aménagement de l'espace et de l'urbanisme. La prégnance des thématiques relatives à la qualité du cadre de vie, la question du bien-être, de l'esthétique et de l'ambiance, mais aussi les rapports à la mémoire et/ou à la communauté, à travers le sentiment d'appartenance, l'ancrage territorial ou l'attachement aux lieux, nous renseignent sur l'importance que revêtent aujourd'hui, dans et pour nos sociétés, les dimensions liées à la sensibilité et à l'affectivité. Dans ce contexte, l'idée que la relation sensible à l'espace n'est pas neutre, que les affects - ce qui nous touche - deviennent une dimension opératoire et donc un enjeu de connaissance sur la relation de l'être humain à son environnement, nous pousse ainsi à évaluer l'importance de ce rapport d'ordre affectif à l'espace. La sociologie (Simmel,
[1903] 2007 ; Ledrut, 1973), la géographie (Dardel, [1952] 1990; Tuan, [1974] 1990), ou encore la psychologie environnementale (Noschis, 1984; Altman et Low, 1992; Scannell et Gifford, 2010) ont contribué à travers des approches différentes, et des choix méthodologiques inhérents à leurs disciplines, à démontrer l'existence de cette relation affective, participant en cela de sa qualification. De ce point de vue, si l'on s'accorde sur le fait que la dimension spatiale renvoie à une réalité concrète avec laquelle les individus entretiennent des relations sensibles, que l'espace n'apparaît pas comme un objet de pure représentation mais qu'il peut nous « affecter » profondément (Ledrut, 1973), il nous semble que les affects interrogent aussi la logique d'action propre à la transformation des espaces habités. L'évaluation de la dimension affective du rapport à l'espace doit se faire autant en termes de compréhension et d'analyse des phénomènes spatiaux, de coordination et de mise en œuvre de l'action collective, qu'en termes d'impact, et de capacité de l'aménagement et de l'urbanisme, à proposer, à suggérer, à accompagner, à orienter l'organisation des territoires et des modes de vie.

Pour autant, la pratique de l'aménagement et de l'urbanisme n'accorde encore que relativement peu d'importance à cette dimension affective. Rarement l'on s'intéresse aux émotions comme une ressource permettant de comprendre les attentes, les besoins, les jugements, leur construction, ainsi que la réponse qu’y apportent les professionnels de l'aménagement, alors qu'elles participent pour une part non négligeable à l'évaluation de l'action et donc directement à sa structuration et à sa mise en œuvre. Les aménageurs apprennent, pour la plupart, à considérer les (leurs) émotions comme une source de distorsion, responsable d'un certain nombre d'erreurs de jugement. Comme le souligne Hoch (2006), l'on trouve un argument principal qui justifie cette conception de l'aménagement et de l'urbanisme impliquant une forme de condamnation des affects. Celle-ci tient à l'influence de la conception rationaliste de l'action qui relègue les émotions et les sentiments à un rôle inférieur en matière de jugement, et ainsi nous ramène au débat ancien - et dépassé (Livet, 2002) - entre raison et passion. Du point de vue de cette conception, souvent dite «dure » ou «froide», les émotions et les sentiments amoindrissent la qualité et l'exercice des facultés intellectuelles. D'ailleurs, 
une part des professionnels reflète et véhicule cette position à travers l'affirmation d'une posture scientifique, experte, fondée en raison et laissant, si possible, le moins de place à des données considérées comme «floues », «molles », « chaudes ». Chez les principaux partenaires de l'action, élus ou habitants, la représentation de l'intervention sur l'espace qui en découle est celle d'une pratique d'abord rationnelle, ou du moins devant nécessairement y tendre de façon privilégiée et presque exclusive, et cela même si cette lecture est régulièrement remise en cause (Novarina, 2004; Hoch, 2006 ; Feildel, 2010). Selon cette conception, il s'agit d'extraire la donnée émotionnelle de l'observation, et de la représentation qui en découle, d'en limiter les potentiels biais, et ainsi de focaliser la technique exclusivement sur la qualité des jugements considérés comme étant dès lors pleinement scientifiques. À cela Hoch oppose un constat issu de l'observation des professionnels de l'aménagement et de l'urbanisme. Le praticien use en réalité du principe de rationalité, et du modèle scientifique qui lui est sous-jacent, davantage comme d'une excuse, d'une rhétorique qui légitime son statut de professionnel expert, que comme d'un réel guide pour l'action (Hoch, 1994). Dans les faits, l'aménageur est un acteur « émorationnel »(Laflamme, 1995; Bouchard, 2006). Il n'est pas différent de tous les autres acteurs et sa pratique, notamment lorsque celle-ci s'insère dans un cadre collectif, ne répond pas seulement à des exigences purement rationnelles. Cette pratique est imprégnée de la complexité des multiples interactions auxquelle l'aménageur a à faire face et se nourrie à la fois de raisons et d'émotions. Les intentions portées par les projets ne se dessinent pas seulement dans le préalable à l'action, mais aussi dans l'interaction, en fonction notamment de la capacité de l'émotion à orienter, à intervenir, à qualifier la relation, à émerger de celle-ci et, de fait, à structurer en retour le processus projectif.

D’ailleurs, les acteurs de l'aménagement le reconnaissent au moins implicitement (Feildel, 2010), bien qu'ils n'en maîtrisent pas consciemment tous les ressorts, puisqu'ils emploient la rhétorique affective principalement à des fins communicationnelles, afin de susciter l'adhésion au projet ou encore afin d'encourager les populations habitantes à prendre part à la vie locale. En effet, il n'est pas rare de trouver la sémantique affective instrumentalisée de la sorte, à l'image de ces slogans mis en avant par certaines municipalités : «J'aime mon quartier, j'en prends soin! » (Montréal en 2004), « J'aime mon quartier et je le prouve! » (Lyon en 2008), et qui ont tendance à se diffuser à mesure que les pratiques communicationnelles prennent de plus en plus de place dans les projets d'aménagement et d'urbanisme (Bailleul; Devisme, 2009). En outre, cette façon qu'ont les acteurs et les institutions de jouer sur la corde sensible n'est pas nouvelle, et dans certains cas, elle peut même être considérée comme un ressort particulièrement puissant - et non moins discutable - dans la construction et l'affirmation des territoires (Genestier, 2009). Mais le pouvoir de persuasion et de motivation de l'émotion, bien connu depuis Aristote, n'est pas seulement manifeste dans le domaine communicationnel, il touche aussi plus directement à l'action et à la logique même de l'aménagement, dans sa dimension projective. Pour Ben Anderson et Adam Holden, plus encore qu'une ouverture possible pour la redéfinition des principes de l'aménagement, la dimension affective est déjà une réalité à l'œuvre dans les pratiques de transformation des espaces, bien qu'elle demeure un impensé scientifique. Ainsi, Anderson et Holden (2008) proposent de qualifier d'« Affective urbanism ", les dynamiques de transformation des espaces qui accompagnent les politiques publiques d'envergure, comme la régénération des centres urbains dans le cadre du programme « capitale européenne de la culture » (Parlement Européen et Conseil de l'Union Européenne, 1999), au titre que la démarche urbanistique impulsée par ces grands évènements est essentiellement animée par un vocabulaire conceptuel spécifique à la logique des affects et des émotions.

L'on trouve également les affects dans un autre registre de l'action publique sur l'espace, qui concerne quant à lui les mobilisations citoyennes autour des projets d'aménagement, que celles-ci soient suscitées ou encouragées par l'institution porteuse du projet, ou alors qu'elles s'érigent spontanément en opposition à cette dernière. En effet, alors que la norme de rationalité est communément tenue pour supérieure dans le cadre des débats et des discussions pour aboutir à la décision publique (Livet, 2007) - l'aménagement des espaces ne faisant pas exception - l'on observe pourtant, à partir de l'étude de ces cas particuliers impliquant une transforma- 
tion substantielle des espaces de vie, la montée en puissance du registre affectif comme régime de justification propre non seulement à structurer les débats et donc à influer sur l'action collective, mais aussi à structurer les représentations et à influer sur les pratiques de l'espace (Feildel, 2011). Pour Lolive, qui a participé à la mise en lumière de ces phénomènes, et qui a souligné notamment l'importance des émotions dans leur capacité à cimenter, à fédérer, des collectifs producteurs de territorialités (Lolive, 1999), il s'esquisse ici une nouvelle problématique d'« esthétisation des espaces publics » (Lolive, 2006). Le fait que le jugement esthétique s'immisce dans les conflits et les controverses autour de l'aménagement des espaces, au point de devenir progressivement un élément central des débats, interroge d'une manière totalement renouvelée la conduite de l'action publique et la place accordée jusqu'ici aux affects.

\section{LES AFFECTS : UN ENJEU CONCEPTUEL ENTRE SENTIR ET RESSENTIR}

L'on constate ainsi un intérêt grandissant de la recherche scientifique, et plus particulièrement des sciences de l'action sur l'espace, pour cette dimension affective, à la mesure certainement du dédain que lui a opposé la perspective rationaliste, ou du moins son interprétation dogmatique. Dès lors, comme nous avons pu le suggérer, cet intérêt se manifeste à travers deux constats relativement simples. D'une part, les habitants ou les usagers, ceux qui vivent l'espace, ceux qui se l'approprient, s'y identifient, ceux-là mêmes qui font l'expérience de cette dimension du vécu, sentent et ressentent, se trouvent affectés par leurs espaces de vie. Ils n'y sont la plupart du temps pas indifférents, ils les éprouvent. En même temps, les pratiques de ces habitants affectent les espaces. Ils produisent un effet non négligeable sur ces espaces, leur conférant un sens particulier, leur donnant une certaine épaisseur. D'autre part, les aménageurs et les urbanistes voient surtout dans l'affectivité un élément de distorsion, bien que paradoxalement ils s'arrogent également, plus ou moins explicitement, cette dimension, à travers notamment les discours qui accompagnent les artefacts de la production urbaine (plans, projets, etc.). Ils usent de la dimension per- formative de l'affectivité, du pouvoir de l'affect, en s'appuyant sur l'émotion, le sentiment - au risque parfois de vider le phénomène de sa substance pour tout simplement emporter l'adhésion. Ainsi, la pratique nous informe sur la nécessité de traiter de cette question affective dans le cadre des approches géographiques et urbanistiques. Pour autant, on constate également un certain flou quant à l'usage et au traitement de ces catégories que sont la sensibilité, la sensation, le sentiment, l'émotion, l'affect. Celles-ci, la plupart du temps, sont traitées sans véritable distinction et sans réelle analytique spécifique.

Le premier travail qui s'impose est celui d'un effort particulier de définition des termes en présence. Mais alors un premier problème apparaît, que Dewey ([1915] 2010) d'ailleurs ne manque de pointer, et que nous rappelons en substance. Pour Dewey, il est aussi indispensable, pour comprendre ce que sont les affects, de discuter et de traiter des facteurs psychologiques, tels que la sensation, la perception, l'intuition, la volonté, l'émotion, que de bien noter le fait que l'ensemble de ces termes ne sont pas employés dans des sens neutres. Au contraire, ils sont lourds de toutes les interprétations que d'ordinaire on leur attache et que leur attachent des traditions fort différentes. Aussi, comme le préconisait Dewey ([1915] 2010), et avant lui encore James ([1884] 2006), la discussion doit assurément se limiter aux traits les plus généraux de ces phénomènes. Le travail consiste donc, dans un premier temps, à la clarification de ces concepts, à l'identification de leurs usages, l'impact que les phénomènes affectifs peuvent avoir au sein même des processus et des logiques de production de l'espace, et réciproquement à l'effet des processus et des formes que revêt cette production, sur les manières de sentir et de ressentir des individus et des groupes sociaux.

À la suite du philosophe Richir (1993), par affectivité, nous proposerons d'entendre en un sens élémentaire ou premier, l'aptitude à être touché, ou en partant de l'origine latine du terme afficere, littéralement "agir sur ", et ad-facere, "vers le faire ", une modification ou un changement se produisant dans le corps en même temps que dans le mental et qui nous pousse à agir, à réagir. À ce stade, nous pouvons d'ores et déjà souligner l'ambivalence de ce terme qui désigne tout à la fois le pouvoir d'être affecté, autrement dit la faculté de sentir, et le sys- 
tème des affects, qui renvoie pour sa part aux différentes dimensions du ressentir, aux phénomènes émotionnels, aux sentiments, aux passions. Sans trop nous appesantir sur ces différents aspects, nous n'en retiendrons pas moins dans cet effort de clarification, que l'affectivité, bien qu'étroitement liée à la question des sens, de la sensorialité et de la sensibilité, doit d'un point de vue strictement méthodologique s'en distinguer. Plus précisément, il nous semble nécessaire de spécifier deux caractéristiques du lien, et en même temps de la distinction, entre sensibilité et affectivité. La sensibilité renvoie à deux dimensions spécifiques bien qu'en réalité indissociables. L'une typiquement sensorielle, qui relève de la sensation et des fonctions physiologiques, autrement dit, qui concerne le fonctionnement de l'organisme, sa capacité à réagir, par les cinq sens, aux stimulations, aux sensations, qu'exerce sur nous le monde extérieur - c'est le senti. L'autre dimension de la sensibilité, renvoyant de la sorte au domaine de l'affectivité, concerne quant à elle la dimension psycho-sociologique de l'activité sensible - c'est le ressenti. L'affectivité, en ce second sens, concerne l'activité psychique de l'individu, l'activité par laquelle il est amené à expérimenter, dans une forme d'instantanéité historicisée, l'activité sensorielle de ses organes, et pour laquelle sont mobilisées l'histoire, la culture des individus et des groupes, leurs systèmes de représentations, leurs manières de penser et d'agir. Partant de ces deux dimensions, étroitement liées, nous distinguons la sensibilité comme rapport psychophysiologique, c'est-à-dire dans ce qu'il implique de liens entre, d'une part, l'activité physique ou physiologique, et d'autre part, l'activité psychique ou psychosociologique, de l'affectivité à proprement parler, l'affectivité étant dès lors reconnue comme la faculté d'éprouver un ensemble d'états dits affectifs : tels que les émotions, les sentiments, etc.

Pour donner une image plus précise de cette distinction et de cette articulation entre la faculté sensorielle et la manière d'en être affectée, ou entre le sentir et le ressentir, nous reprendrons volontiers l'idée de la «représentation redoublée » de Foucault (1966). Considérant la représentation et l'évolution des manières scientifiques de comprendre ce que « représenter » implique, Foucault nous dit « la nature humaine se loge dans ce mince débordement de la représentation qui lui permet de se re-présen- ter » et « toute la nature humaine est là : juste assez à l'extérieur de la représentation pour qu'elle se présente à nouveau, dans l'espace blanc qui sépare la présence de la représentation et le "re-" de sa répétition » (Foucault, 1966). Entre sentir, phénomène sensoriel, et ressentir, phénomène de l'affectivité, se joue en quelque sorte le même enjeu, c'est dans cet espace blanc du « re-sentir» que se loge, comme le notait Dewey, «la contribution humaine » à cette expérience du sentir. « Le soi agit et subit, et ce qu'il subit ne consiste pas en impressions qui s'inscrivent dans une cire inerte mais dépend de la manière dont l'organisme réagit et répond. Il n'est pas d'expérience où la contribution humaine ne soit pas un facteur responsable de ce qui se produit réellement. L'organisme est une force, il n'est pas qu'une pellicule sensible» (Dewey, [1915] 2010).

À la stricte instantanéité de la sensibilité, réponse immédiate à une perception du fait stimulant, nous accordons à l'affectivité cette spécificité de dépendre de l'expérience humaine et donc de s'incarner, au moins en partie, dans une perspective temporelle élargie. Une des caractéristiques qui permet ainsi de distinguer la sensibilité de l'affectivité tient à la dimension temporelle de ces phénomènes. Certes l'on pourrait opposer à cette conception que l'émotion, cet état affecté que tout un chacun expérimente, s'exprime dans la majorité des cas dans l'instant où l'évènement qui la suscite se produit - d'ailleurs l'on associe volontiers la surprise à l'émotion, et dans le sens commun l'émotion est généralement brusque et momentanée - néanmoins l'émotion se distingue de la sensibilité qui en est la cause. L'émotion dans son instantanéité propre, n'est jamais déconnectée d'un vécu qui engage l'individu dans son histoire présente, la situation où il est amené à éprouver, mais aussi son expérience passée, les émotions qu'il a précédemment pu éprouver, la projection d'un futur, un horizon d'attente. Sensibilité et affectivité se distinguent au moins sur ce point, la perspective temporelle qui leur est propre. En ce sens, tout état affectif, aussi bref, rapide, abrupt soit-il, se distingue de la sensibilité qui en est la cause, dans la mesure où cette dernière ne semble pas engager la profondeur de la dimension temporelle dans ce qui devient au-delà d'une stricte faculté - la sensorialité - une véritable manière d'éprouver - l'affectivité. La psychologie environnementale l'a également souligné, la relation 
à un espace donné, y compris dans sa dimension affective - et non pas strictement sensorielle - est au-delà du présent, tributaire du passé de l'individu ainsi que de son futur projeté. "Si l'on se penche sur la relation individu-environnement, la dimension temporelle est omniprésente, elle intervient de différentes manières dans l'ancrage territorial et le bien-être, elle conditionne la manière dont l'environnement est perçu, évalué et les pratiques qui y sont déployées. [...] Se sentir "chez soi" est conditionné par les motivations, le statut social et professionnel, la situation familiale et surtout les projets de l'individu. De même, les lieux ont un passé qui contribue à leur interprétation actuelle, et un futur qui est susceptible de nous guider dans nos actions à travers nos représentations anticipatoires » (Moser, 2003).

Si pour diverses raisons, ces phénomènes - sensibilité et affectivité - sont en réalité difficilement dissociables, il n'en demeure pas moins que l'étude de l'affectivité à proprement parler nécessite de dépasser le strict rapport sensible de l'individu à l'espace, en y intégrant notamment l'horizon temporel spécifique aux individus, avec tout ce que celui-ci comporte d'implications notamment sur les plans sociaux et spatiaux. S'il convient de ne pas négliger cette part de la sensibilité qui nous affecte lorsque nous ressentons, lorsque nous faisons l'expérience de l'espace, pour autant, il ne peut être question non plus de négliger ce qu'implique la prise en compte de l'affect dans une perspective temporelle, à savoir l'inévitable participation des affects à nos manières de sentir, le fait que l'affectivité ne saurait être tenue pour strictement co-originaire à l'individu mais trouve à se déployer lors de la rencontre de son objet. Ainsi, lorsque l'on croit communément que l'émotion est l'expression de l'incarnation subjective d'un phénomène sensible, l'on se trompe pour partie selon Despret (2001). On oublie que l'affectivité est bien plus que cela, « l'expérience affective, les émotions, les sentiments [...] sont [aussi] des outils performatifs de modification des expériences sensibles : opérer un changement de rythme pour se laisser habiter par une expérience, ralentir; accepter de ne plus savoir ce que l'on croyait savoir, hésiter» (Despret, 2001). L'émotion n'est pas seulement une matière première, un donné vierge et malléable, souligne également Boullier. «Elle est aussi un vecteur, une médiation, elle "fait faire" autrement, en ce sens qu'elle nous fait hésiter et nous laisse pénétrer, elle circule, et, étant ainsi distribuée, elle devient un objet contagieux, elle fait aussi sentir en commun » (Boullier, 2010). Pour Deleuze et Guattari ([1991] 2005), « les affects sont précisément ces devenirs non humains de l'homme », ils dépassent l'humain, "ils débordent la force de ceux qui passent par eux », et en même temps ils sont le devenir de l'homme, au sens où justement l'on passe par eux, « on n'est pas dans le monde, on devient avec le monde».

Les individus sentent et ressentent, les groupes produisent et partagent des affects, les sociétés construisent et, en quelque sorte, obligent à des manières de sentir. Dans ce système, les villes, la forme que prend l'espace urbanisé, et plus généralement les organisations spatiales, ainsi que leurs modes de production, ne sont pas neutres et sont à prendre autant comme objet de l'expérience - ce que nous sentons - que comme créateurs d'affects en devenir, générateurs de manières de sentir. Bien que la relation soit tangible entre l'homme et son environnement, il n'est pas pour autant question d'établir un lien de détermination directe entre l'espace et l'affect, entre l'objet et le sujet. Si certains s'y sont risqués, comme Simmel ([1903] 2007) dans un court essai sur « les grandes villes et la vie de l'esprit », ce que l'on retient avant tout c'est l'intuition programmatique qui nous enjoint à considérer de concert, dans un mouvement de co-construction, l'agencement spatial des sociétés et l'expérience affective en tant qu'ils sont mutuellement produits et producteurs. Il faut comprendre, comme le souligne Dewey ([1915] 2010), que l'interaction entre «sujet » et «objet » est si étroite, si permanente et indissociable, la consubstantialité de l'un et de l'autre est si vraie et si complète, qu'il importe finalement assez peu de savoir lequel de ces deux facteurs prédomine dans l'interaction, mais bien davantage de comprendre comment tous les deux interagissent, comment l'un alimente l'autre et réciproquement. C'est précisément cela l'affectivité, selon Ledrut (1973), ce qui établit « un lien entre le sujet et l'objet, tel que le sujet apparaît affecté dans son être ».

Dès lors, il convient de souligner que la sensation, ce que l'espace fait en nous, et le ressenti qui l'accompagne, autrement dit ce qu'il évoque, ne sont pas indépendants, l'un et l'autre ne se plaçant pas dans un rapport de causalité mais bien davantage de quasi-unicité. Ainsi, l'on ne saurait faire de l'af- 
fectivité la résultante d'une sensibilité particulière, tant l'affectivité explique, détermine et interagit de façon étroite avec nos manières d'être sensibles. Il s'agit en réalité de comprendre comment le sentir et le ressentir, l'espace et l'affect, sont impliqués dans une relation de co-construction.

\section{UNE MÉTHODE POUR APPROCHER LA DYNAMIQUE AFFECTIVE DU SENTIR}

Poursuivant cette volonté d'analyse du système des affects, tout à la fois producteur et produit d'organisations socio-spatiales, nous proposons une méthode à la jonction de deux types d'approches, géographique et biographique. Pour mieux comprendre ces modalités affectives de la relation à l'environnement et, plus généralement, tout ce qui fait la manière pour les individus d'éprouver leurs espaces de vie, nous proposons de procéder selon une approche reposant sur l'utilisation de l'objet cartographique comme outil permettant, d'une part, la prise de conscience d'un vécu géographique d'ordinaire difficilement préhensible et, d'autre part, de façon étroitement liée à cette prise de conscience première, comme outil de réactivation et d'approfondissement de la composante affective de ce vécu spatial (Martouzet et al., 2010).

La démarche méthodologique (figure 1) que nous proposons répond à cette volonté, propre au domaine des affects, d'appréhender le rapport affectif à l'espace comme un construit à la fois individuel et social, dans une perspective temporelle et spatiale élargie, autrement dit, à l'échelle de l'ensemble de la vie des acteurs spatiaux, en privilégiant la parole pour atteindre la couche plus ou moins conscientisée des affects (Cahour, 2006). La méthode en question s'articule autour de deux temps : un « récit de vie spatialisé » et une " herméneutique cartographique » (Feildel, 2010). À la suite d’un « récit de vie spatialisé » réalisé avec l'interviewé, nous exploitons dans un second entretien la fonctionnalité « transitionnelle » d'un " objet créé et donné » (Winnicott, [1971] 1975), en l'occurrence la représentation cartographique de la spatialité de l'individu à l'échelle de sa vie, dans le but de dépasser les traditionnelles limites du récit de vie, comme présentation acceptable de soi et par soi (Bourdieu, 1986), pour atteindre in fine les dynamiques affectives. Consistant en une épreuve de qualification des dimensions spatiales de l'existence, cette seconde étape appelée « herméneutique cartographique ", réalisée par l'interviewé lui-même, accompagné dans cet exercice d'auto-analyse (Bourdieu, 1993; Hoyaux, 2006) provoqué par l'enquêteur, nous permet de constater l'importance des dimensions affectives dans la représentation individuelle et sociale de l'espace. Ainsi, l'on observe à travers le discours produit, à côté des modalités traditionnelles du rapport à l'espace (de types fonctionnelles, économiques, sociales, etc.), une modalité affective non moins primordiale dans la relation qu'entretient l'individu avec son milieu. L'analyse couplée de ces

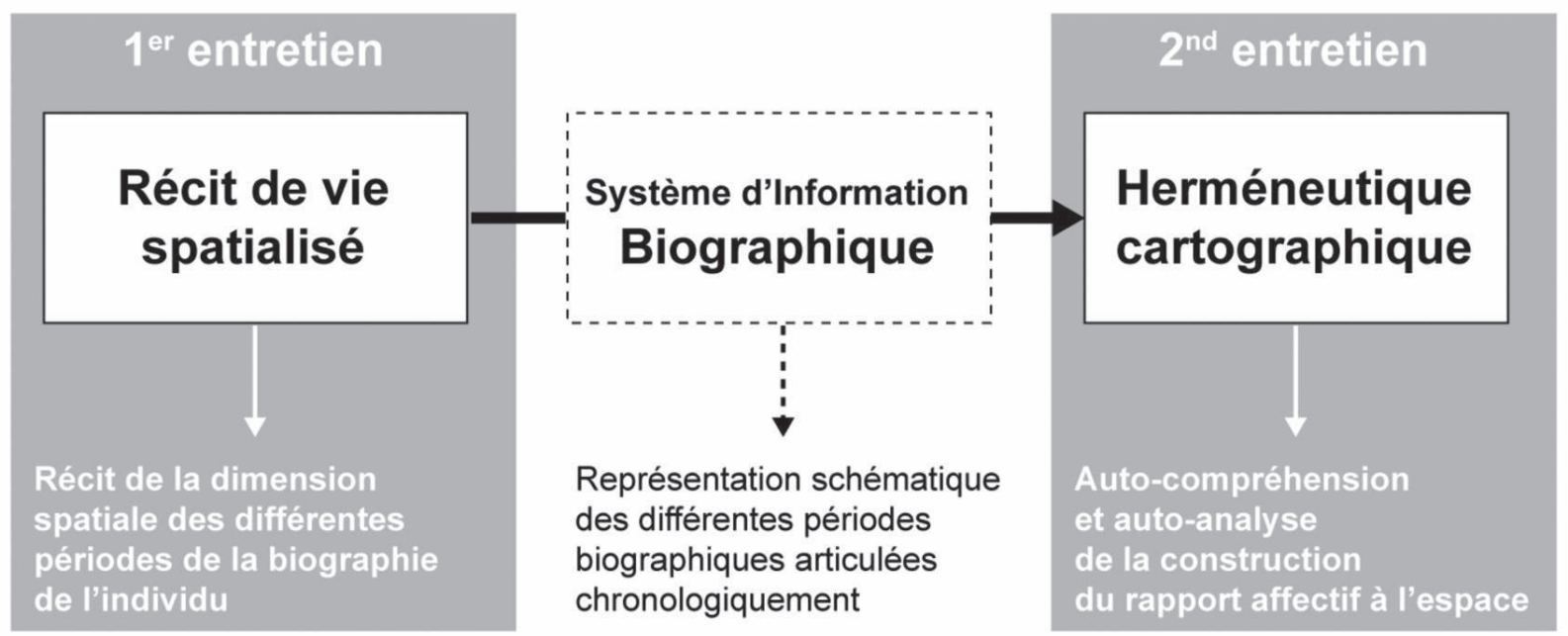

Figure 1 : Récit de vie spatialisé, système d'information biographique et herméneutique cartographique Life spatial history. Biographical Information System and cartographic hermeneutics 
deux entretiens permet dès lors une mise en lumière de l'agencement des liens matériels et affectifs aux différents espaces de vie : comment se forme le potentiel d'affectivité des espaces de vie des individus. Et comment, par là-même, s'établit dans la relation à ces lieux, la dialectique entre sensibilité et affectivité, entre le sentir et le ressentir. Quelle influence elle opère sur la représentation et la pratique des lieux géographiques.

Concrètement, nous nous sommes entretenus avec un certain nombre d'habitants rencontrés et sélectionnés dans différents contextes socio-spatiaux. Nous leur avons demandé à chacun de réaliser, dans un premier temps, un récit de leur vie, en leur demandant systématiquement de préciser et de décrire la dimension spatiale caractéristique des différentes périodes de leur biographie. Ensuite, nous avons cartographié ce récit en adoptant pour cela un code géométrique volontairement simple, facilement compréhensible pour tout un chacun et éminemment lié à la symbolique spatiale, des symboles circulaires pour les lieux et des traits pour les mobilités (Lévy, 2008). Le résultat de ce travail est la production d'une représentation schématique, une carte, ou plutôt un ensemble de cartes, articulées chronologiquement dans un « Système d'Information Biographique »(Feildel, 2010, 2012), reprenant trait pour trait le récit de vie spatialisé que nous avait délivré l'individu en première intention. Les différentes cartes (figure 2 - planche IV) ainsi réalisées nous ont d'abord permis d'appréhender, sous certaines conditions, les logiques spatiales propres aux parcours de vie des individus interrogés.

Notre démarche ne s'est cependant pas limitée à ce premier résultat. La carte a été envisagée comme le support privilégié de l'approfondissement des dimensions existentielles du récit spatial délivré par l'individu. En accord avec notre objectif qui était de comprendre le processus d'appropriation subjective de la dimension spatiale, autrement dit, pour approcher la relation affective qui fait que nous habitons les espaces de nos vies et que, réciproquement, ces espaces nous habitent, une seconde étape de notre enquête a résidé dans la mise en œuvre de l'épreuve d'« herméneutique cartographique » pour laquelle il a été demandé à l'individu d'interpréter sa propre carte.

L'un des avantages que permettait l'utilisation de la carte comme support herméneutique était son caractère intrinsèquement faux, imparfait, insuffisant. La «pauvreté » de la carte a alors été la source et l'origine pour l'interviewé d'un approfondissement des dimensions de son parcours spatial de vie (Martouzet et al., 2010). Notamment, la confrontation entre la matérialisation symbolique du récit (l'individu faisant confiance au travail d'expression symbolique du cartographe) et le souvenir des éléments de ce récit par l'interviewé a permis une remise en question de l'organisation première du récit, une déconstruction de la logique énoncée par l'interviewé lui-même. De cette phase d'autocompréhension (Brubaker, 2001) et d'auto-analyse, il a tout d'abord émergé l'évidence du caractère lissé du « récit de vie spatialisé » délivré en première instance. Ensuite, la carte a permis d'ouvrir le discours de l'interviewé sur le champ, plus vaste, des référents spatiaux de l'individu - et en particulier leur dimension affective. Cette dimension affective du rapport de l'individu à l'espace a notamment été approfondie à travers un exercice de qualification, de notation des composantes spatiales de la trajectoire de chaque interviewé. Le résultat de cette épreuve de qualification, le nouveau discours qui a accompagné l'« herméneutique cartographique », a été le véritable objet, l'élément central de notre analyse, ce qui a permis de révéler l'importance de la dimension affective, comment celle-ci renvoie à un construit historique et situé, qui informe la manière d'éprouver la dimension spatiale de l'existence. C'est ce qu'illustrent les citations reproduites ci-après, concernant un même espace raconté par un enquêté aux deux étapes du protocole, pour la première, dans le cadre de son récit de vie spatialisé, et pour la deuxième, de son épreuve d'herméneutique cartographique :

"Donc point origine : évidemment toujours la naissance, donc première année de ma vie, en fait je suis né à Pithiviers en pleine Beauce, mais mes parents habitaient à Orléans, où ils ont dî vivre un an... Je n'en ai absolument aucun, aucun souvenir. Alors, ils habitaient à Orléans, et ma mère est allée accoucher à Pithiviers parce que c'était une clinique qui était censée être un petit peu avant-gardiste avec une technique d'accouchement sans la douleur [...]. Mais en tout cas un an à Orléans dont je n'ai aucun souvenir. » (Récit de vie spatialisé de L2) 
"Ça m'arrive de repasser à Pithiviers et je trouve que cette ville est à la fois très triste et... mais ça me fait quelque chose de passer dans un endroit où je sais que je suis né. Et... je suis toujours attaché à Pithiviers, ma madeleine de Proust. À savoir que d'une certaine manière... bon on est dans l'ordre de l'affectif, mais ma mère m'a toujours... m'a toujours dit t'es né à Pithiviers, à Pithiviers y'a un super gâteau et j'ai toujours adoré les pithiviers. Et quand je passe à Pithiviers en général on s'arrête acheter un pithiviers dans cette ville qui est plutôt une petite ville. C'est pas très gai, c'est plutôt populaire. Et c'est sûr que c'est pas une ville qui serait marquante pour moi, si y'avait pas cette mémoire. Donc effectivement, si je me réfère à la période de ma naissance c'est du neutre, si je la qualifie depuis aujourd'hui c'est pas tout à fait neutre. » (Herméneutique cartographique de L2)

\section{LE RESSENTIR «AVANT » LE SENTIR}

Qu'en est-il ressorti plus généralement? Sans pouvoir entrer plus en détail dans l'analyse du matériau ainsi produit, l'on notera toutefois que la méthode autorise l'appréhension, et la compréhension, des différentes dimensions imbriquées dans la constitution et l'expression du rapport affectif à l'espace.

Nous avons pu appliquer cette méthode à plusieurs terrains d'études dans l'agglomération de Tours ${ }^{1}$, interrogeant pour cela une trentaine d'habitants localisés dans la périphérie plus ou moins proche du centre urbain, et vivant dans des espaces soumis à différentes logiques urbanistiques : un centre ancien consolidé, un quartier nouvellement construit, un espace périphérique en voie de densification. Bien que relativement restreint par le nombre - la diversité a pour nous primé sur la représentativité sociale des personnes interrogées - l'échantillon n'en a pas moins permis d'appréhender une certaine diversité socio-spatiale, puisque le «terrain d'étude » particulier qu'impliquait la méthode, mise en œuvre à l'échelle biographique, a couvert un ensemble de lieux géographiques beaucoup plus large que l'espace localisé dans l'instant

1. Pour plus de détails sur les modalités de mise en œuvre et la portée de l'enquête réalisée sur les différents terrains d'études tourangeaux, nous renvoyons le lecteur à Feildel B., 2010, Espaces et projets à l'épreuve des affects. Pour une reconnaissance du rapport affectif à l'espace dans les pratiques d'aménagement et d'urbanisme, Thèse de doctorat en aménagement et urbanisme, Université François-Rabelais, Tours, 651 p. présent de l'enquête. En effet, au total ce sont plus de 1200 lieux qui ont été mentionnés par les habitants, appartenant indifféremment aux registres du réel, du symbolique ou de l'imaginaire, mais construits pour chacun dans une même réalité discursive. Finalement, la richesse du discours délivré a permis d'éclairer la façon dont se construit le rapport affectif au fil du temps, au fur et à mesure des grandes étapes de la vie, et son influence dans l'instant présent sur la nature de l'éprouvé. À titre d'illustration seulement - notre propos n'étant pas ici d'entrer dans une analyse fine et détaillée des processus à l'œuvre - nous reprendrons trois extraits des discours que nous ont délivré les enquêtés, au cours de leur herméneutique cartographique. Ces extraits font une large part aux manières de sentir et à l'influence du rapport affectif, à son inscription dans le temps long, en vue notamment de la détermination de possibles choix de localisation spatiale.

"La forêt, alors là c'est du côté de la famille de mon père, le village natal de mon père. Donc la forêt de Rosselange, c'est très très important et ça le reste. Un lieu de ressourcement. Parce que c'est lié à ma famille. Parce que ça a toujours été la plus jolie, et parce que mon grand-père y faisait du bois, voilà c'est lié à tout ça. Et comme je n'ai pas connu mon grand-père, c'est peut-être aussi une manière d'aller dans les lieux où il a passé beaucoup de temps. Ony allait le dimanche, avec les parents, courir, regarder les arbres, les animaux qui passent. Ça fait pas très longtemps que l'on est à Tours, mais dans les autres lieux que j'ai habités, la forêt c'est quelque chose que j'ai recherché. Et quand c'était pas là, et bien cela m'a manqué. Un sentiment de manque. Alors là je ne peux pas déterminer si c'est vraiment un besoin ou si c'est simplement une envie. En tout cas, quand il n'y a pas de forêt, il me manque quelque chose. " (Herméneutique cartographique de P1)

"Là c'est un des seuls endroits où je me sens... je me sentais pas bien dans cet appartement, dans cet environnement. C'était au bout du boulevard Heurteloup cette rue, et elle dormait. Elle donne sur les quais de la Loire, vers le vieux Saint-Pierre, mais au moment où j'y ai vécu, à cette période-là, c'était pas un endroit qui me reflétait. Si j'y vivais maintenant oui, mais à cette époque-là ça ne bougeait pas, 
c'était pas mon truc. Ça ne me correspondait pas. " (Herméneutique cartographique de S2)

"Alors j'ai eu envie d'habiter dans le quartier des Deux-Lions, parce que d'abord j'aime bien ce coinlà. J'aime les bords de rivière. J'aime bien habiter au bord d'une rivière. Alors la Loire ou le Cher? La Loire, à part Rochecorbon... à Saint-Cyr-surLoire, les bords de Loire, il y a quand même la route qui gêne et puis ce n'est pas forcément très agréable donc on a plutôt visé le Cher. Et les Deux-Lions parce qu'on y est allés... un jour on est venus se balader avec mon mari, je crois qu'on avait dî aller au cinéma... et puis on s'est baladés jusqu'au bord $d u$ Cher. On est tombés sur ces maisons en bois de toutes les couleurs qui étaient en construction. On est rentrés dedans, et en fait ça a démarré comme ça. J'aime bien le bord du Cher. Je trouve que c'est, enfin que c'était joli à l'époque... donc je trouvais que c'était sympa... c'était un peu la campagne en ville. » (Herméneutique cartographique de G1)

La sensibilité, loin d'être entièrement déterminée par le lieu et par la situation présente, nous est plutôt apparue comme le résultat d'un dosage complexe, mettant en jeu à la fois la capacité du lieu à imposer son ressenti mais aussi la force des liens, c'est-à-dire l'ensemble des relations aux différents lieux de vie pouvant potentiellement être mobilisé et actualisé pour orienter le sentir. Ainsi, nous avons pu observer concrètement cette tension permanente entre l'espace et l'individu, l'importance de la nature relationnelle et situationnelle du ressentir, et en même temps, la plus ou moins grande unité dans laquelle les éléments du milieu sont susceptibles de guider les individus vers un éprouvé commun ou partagé. Le sentir, pour autant qu'il nous soit donné, ne trouve sa réelle expression et sa réelle utilité que dans le ressentir. Le rapport affectif ne peut pas être donné ou seulement induit par l'objet, il peut seulement s'accomplir dans l'interaction entre le sujet et l'objet. Les lieux et les milieux de vie n'offrent pas les mêmes «potentialités dispositionnelles » (Jeudy, 2003) ou les mêmes "prises affectives » (Audas, 2011) pour le ressentir. Ils n'offrent pas la même tonalité affective, et par conséquent ne nous guident, ne nous orientent, pas de la même manière dans notre sentir. Encore faut-il comprendre la manière dont les individus et les groupes d'indivi- dus se saisissent de ces potentialités, comment ils prennent prises, comment ils ressentent et dès lors font l'expérience sensorielle de leurs espaces de vie. À proprement parler, les affects ne sont ni endogènes, ni exogènes, ils sont la qualité actualisée dans l'instant présent des multiples formes relationnelles que nous avons, avons eu et auront aux choses, aux objets, aux évènements - l'affectivité nous dispose à des manières de « sentir-ressentir» (Florival, 1995). L'occurrence affective, sa capacité d'émergence, y compris son caractère contagieux, va contribuer à orienter, à focaliser l'attention sur certaines propriétés de la situation, en mettant ainsi les sens en éveil, en faisant sentir. Le sentir n'est pas premier, c'est le ressentir - ce que produit l'affectivité; l'affectivité ne faisant véritablement sens que dans le ressentir (Florival, 1995) - qui guide déjà notre manière de sentir, c'est-à-dire d'éprouver sensoriellement notre environnement. Considérer que le ressentir, la tonalité affective du lieu, autrement dit sa capacité à entrer en résonance avec les valeurs de l'individu, à s'incarner physiologiquement et comportementalement, considérer que l'émotion, le sentiment, les affects sont performatifs, qu'ils nous «font faire ", et en particulier qu'ils nous «font sentir », est primordial. Ce renversement, ou cette réunion « sentirressentir », oblige à changer radicalement de point de vue, à reconsidérer notre façon de concevoir la relation, notamment affective, que chacun entretient avec ses espaces de vie. Ne plus mettre la sensation ou la perception sensible au commencement de toute chose, avant l'impulsion de ce qui nous affecte, et qui en réalité oriente déjà notre percevoir et notre sentir, c'est ainsi considérer que « l'émotion déclenchée est au service de l'état de choses perçu » (Dewey, [1915] 2010). Mais alors, comment le rapport affectif se construit-il, comment s'actualise-t-il dans les différentes situations spatiales que nous traversons, comment nous fait-il sentir? Plus loin, avec les affects, avec la logique qui leur est propre, particulière mais aussi de mieux en mieux connue (Channouf et Rouan, 2002), nous disposons d'un ensemble de connaissances susceptibles d'être intégrées à une réflexion globale sur le sentir et le ressentir, sur la perception et la signification des espaces et sur la construction des atmosphères affectives (Anderson, 2009). 


\section{Conclusion}

Loin d'épuiser la problématique de l'affectivité, et de résoudre la question de la participation des affects à l'agencement spatial des sociétés, la méthode proposée, et la façon dont elle nous informe sur la relation qui s'établit entre sensibilité et affectivité, nous semble cependant une contribution supplémentaire pour l'intégration de la donnée affective en aménagement de l'espace et en urbanisme. Tandis que l'histoire et la sociologie se sont depuis un certain temps déjà emparées de ces questions, sondant nos manières d'être sensibles, leurs évolutions dans le temps et le rôle des sociétés dans leur élaboration, nous en appelons pour notre part à une véritable " géographie de l'affectivité ", capable de répondre à ces questions qui nous paraissent désormais primordiales : comment sensibilités et espacements - tant spatiaux que temporels - se construisent réciproquement pour organiser l'espace des sociétés? Quels sont les processus de spatialisation de l'affect et comment ils participent d'une expression territorialisée, à la fois historique et géographique, individuelle et sociale, des sensibilités? Plus loin encore, c'est bien ici la question des affections qui est au cœur, en particulier la place de l'affectivité dans les théories de l'action sur l'espace. De fait, il s'impose également à l'aménagement et à l'urbanisme de se saisir de ces questions, et de ménager une place pour les affects dans le cadre de la réflexion sur les pratiques intentionnelles et les modalités procédurales de la transformation des espaces habités. Alors que l'aménagement durable des territoires, et les modalités de sa mise en œuvre à travers notamment la plus grande intégration des différents publics au sein des démarches de projet, fait figure aujourd'hui de paradigme dominant en aménagement et en urbanisme, qu'en est-il de la prise en compte des manières de sentir? Comment et dans quelle mesure les différentes formes d'organisations spatiales, des espaces périurbains aux «éco-quartiers », nous affectent et participent de nos manières d'être sensibles? Quelles sensibilités mettent en avant des modes d'action, des manières de penser et d'agir, qui peinent encore à intégrer une donnée sensible pourtant profondément structurante? Une conception renouvelée de l'action sur l'espace, prenant en compte la dimension affective, se doit désormais de reconnaître ce qui relie les indi- vidus, les habitants, les usagers, les professionnels de l'aménagement à l'espace. De fait, l'ambition d'un urbanisme affectif ne peut plus simplement se cantonner à l'aménagement de l'espace des lieux, mais doit aussi considérer le ménagement les liens que chacun tisse avec son environnement.

\section{Remerciements}

L'auteur tient à remercier les différents relecteurs de l'article, depuis sa présentation au congrès de la Société Internationale d'Ethnologie et de Folklore de 2011 jusqu'à la présente publication, pour leurs suggestions et leurs commentaires toujours constructifs.

\section{Bibliographie}

Altman I., Low S. M., 1992. Place attachment, New York, Plenum Press, $314 \mathrm{p}$.

Anderson B., 2009. Affective atmospheres, Emotion, Space and Society, Vol. 2, n ${ }^{\circ}$ 2, p. 77-81.

Anderson B., Holden A., 2008. Affective Urbanism and the Event of Hope, Space and Culture, Vol. 11, n 2, p. 142159.

Audas N., 2011 1. La dynamique affective envers les lieux urbains: la place des temporalités individuelles et urbaines, Thèse de doctorat en aménagement de l'espace et urbanisme, université F. Rabelais, 511 p.

Bailleul H., 2009. Communication et projets urbains. Enjeux et modalités de la communication entre acteurs du projet et habitants, Thèse de Doctorat en aménagement de l'espace et urbanisme, université F.-Rabelais - Tours, 589 p.

Bigando E., 2006. La sensibilité au paysage ordinaire des habitants de la grande périphérie bordelaise (communes du Médoc et de la Basse Vallée de l'Isle), Thèse de doctorat en géographie, université Michel-de-Montaigne - Bordeaux 3, 490 p.

Bochet B., Racine J.-B., 2002. Connaître et penser la ville : des formes aux affects et aux émotions, explorer ce qu'il nous reste à trouver. Manifeste pour une géographie sensible autant que rigoureuse, Géocarrefour, Vol. 77, $\mathrm{n}^{\circ} 2$, p. 117-132.

Bouchard P., 2006. Théorie de l'action et parcours de vie, Nouvelles perspectives en sciences sociales, Vol. 1, $\mathrm{n}^{\circ} 2$, p. 67-114.

Boullier D., 2010. La ville évènement. Foules et publics urbains, La ville en débat, Paris, PUF, PUCA, 147 p.

Bourdieu P. (dir.), 1986. Liillusion géographique, Actes de la recherche en sciences sociales, vol. 62-63, p. 69-72.

Bourdieu P. (dir.), 1993. La misère du monde, Paris, Seuil, 1460 p.

Brubaker R., 2001. Au-delà de l'«identité », Actes de la recherche en sciences sociales, Vol. 139, n² 2, p. 66-85. 
Cahour B., 2006. Les affects en situation d'interaction coopérative : proposition méthodologique, Le travail humain, Vol. $69, n^{\circ} 4$, p. $379-400$.

Channouf A., Rouan G. (dir.), 2002. Émotions et cognitions, Neurosciences \& Cognition, Bruxelles, De Boeck université, $319 \mathrm{p}$.

Damery C., 2008. Espace public, patrimoine et milieu affectif (Exemples du marais d'Orx et du Domaine d'Abbadia), Thèse de doctorat en géographie, université de Pau et des Pays de l'Adour, $501 \mathrm{p}$.

Dardel E., [1952] 1990. L'homme et la terre. Nature de la réalité géographique, Paris, Éditions du CTHS, 199 p.

Deleuze G., Guattari F., [1991] 2005. Qu'est-ce que la philosophie?, Paris, Les Éditions de Minuit, 206 p.

Despret V., 2001. Ces émotions qui nous fabriquent. Ethnopsychologie de l'authenticité, Paris, Les Empêcheurs de penser en rond, $359 \mathrm{p}$.

Devisme L. (dir.), 2009. Nantes. Petite et grande fabrique urbaine, Nantes, Parenthèses, 263 p.

Dewey J., [1915] 2010. L'art comme expérience, Paris, Gallimard, $596 \mathrm{p}$.

Durкheim E., 1895. Les règles de la méthode sociologique, Paris, Félix Alcan, 186 p.

Elster J., 2003. Proverbes, maximes et émotions, Paris, PUF, $182 \mathrm{p}$.

Feildel B., 2004. Le rapport affectif à la ville: Construction cognitive du rapport affectif entre l'individu et la ville, Centre d'Études Supérieures en Aménagement, université F.-Rabelais - Tours, $112 \mathrm{p}$.

Feildel B., 2010. Espaces et projets à l'épreuve des affects. Pour une reconnaissance du rapport affectif à l'espace dans les pratiques d'aménagement et d'urbanisme, Thèse de doctorat en aménagement de l'espace et urbanisme, université F.-Rabelais - Tours, $651 \mathrm{p}$.

Feildel B., 2011. Émotions et participation ou comment la délibération autour des projets d'aménagement participe de la construction du rapport affectif à l'espace, Journée d'études sur les effets de la participation, «GIS Participation du public, décision, démocratie participative », Paris, 21 octobre 2011, [http://www.participation-et-democratie. fr/ sites/default/files/atelier_1-1_benoit_feildel.pdf].

Feildel B., 2012. La carte pour approfondir la dynamique des géographies affectives, Mappemonde, 2, n 106, [http://mappemonde.mgm.fr/mum34/mois/moil2201.html].

Florival G., 1995. Questions d'anthropologie philosophique aujourd'hui, Revue Philosophique de Lowvain, Vol. 93, n 4 , p. 579-596.

Foucault M., 1966. Les mots et les choses. Une archéologie des sciences humaines, Paris, Gallimard, 400 p.

Genestier P., 2009. Aménagement de l'espace, production de sens et identité narrative. Les fonctions identificatoire et institutionnalisante du territoire en question, dans BAUdin G., Bonnin P. (dir.), Faire territoire, Paris, Éditions Recherches, p. 205-225.

Hосн C., 1994. What planners do: Power, politics, and persuasion, Chicago, Planners Press, American Planning Association, 364 p.
Hoch C., 2006. Emotions and Planning, Planning Theory and Practice, Vol. $7, \mathrm{n}^{\circ}$ 4, p. 367-382.

Hoyaux A.-F., 2006. Pragmatique phénoménologique des constructions territoriales et idéologiques dans les discours habitants, L'Espace géographique, Vol. n³, p. 271-285.

James W., [1884] 2006. Qu'est-ce qu'une émotion?, dans James W., Les émotions, Paris, L'Harmattan, p. 25-44.

Jeudy H.-P., 2003. Critique de l'esthétique urbaine, Paris, Sens \& Tonka éditeurs, $165 \mathrm{p}$.

Labussière O., 2007. Le défi esthétique en aménagement : vers une prospective du milieu. Le cas des lignes très haute tension (Lot) et des parcs éoliens (Aveyron et Aude), Thèse de doctorat en géographie, université de Pau et des Pays de l'Adour, 606 p.

Laflamme S., 1995. Communication et émotion. Essai de microsociologie relationnelle, Paris, L'Harmattan, $191 \mathrm{p}$.

Ledrut R., 1973. Les images de la ville, Paris, Anthropos, 386 p. LÉvy J., 2008. Échelles de l'habiter, Paris, PUCA, 368 p.

Livet P., 2002. Émotions et rationalité morale, Paris, PUF, 291 p.

Livet P., 2007. Émotions et révision : la dynamique des débats, dans Revel M. et al. (dir.), Le débat public : une expérience française de démocratie participative, Paris, La Découverte, p. 339-352.

Lolive J. (1999), Les contestations du TGV Méditerranée. Projet, controverse et espace public, Paris, L'Harmattan, 314 p.

Lolive J. (2006), Des forums hybrides à l'esthétisation des espaces publics, Cahiers de géographie du Québec, Vol. 50, $n^{\circ} 140$, p. 151-171.

Lussault M., Sтоск M., 2010. « Doing with space » : towards a pragmatics of space, Social Geography, Vol. 5, $\mathrm{n}^{\circ} 1$, p. 11-19.

Manola T., 2012. Conditions et apports du paysage multisensoriel pour une approche sensible de l'urbain, Thèse de doctorat en aménagement de l'espace et urbanisme, université Paris-Est, 646 p.

Martouzet D., 2002. Le rapport affectif à la ville : conséquences urbaines et spatiales, le cas de Fort-de-France, Annales de Géographie, ${ }^{\circ}$ 623, p. 73-85.

Martouzet D., 2007. Le rapport affectif à la ville : positionnement théorique et épistémologique, Praxis, Revue électronique d'Aménagement, [http://www.revue-praxis.fr/document. php?id=117].

Martouzet D., Bailleul H., Feildel B., Gaignard L., 2010. La carte : fonctionnalité transitionnelle et dépassement du récit de vie, Nature, Sciences, Sociétés, Vol. 18, n² 2, p. 158170 .

Moser G., 2003. Questionner, analyser et améliorer les relations à l'environnement, dans Moser G., Weiss K. (dir.), Espaces de vie. Aspects de la relation homme-environnement, Paris, Armand Colin, p. 11-42.

Noschis K., 1984. Signification affective du quartier, Paris, Librairie des Méridiens, 170 p.

Novarina G., 2004. Les nouvelles modalités de l'analyse urbaine : décrire plutôt que prescrire, dans Chalas Y. (dir.), L'imaginaire aménageur en mutation, Paris, L'Harmattan, p. $59-77$. 
Parlement Européen et Conseil de l'Union Européenne, 1999. Capitale européenne de la culture, Journal officiel des Communautés européennes, L 166/1, [http://eur-lex.europa. eu/LexUriServ/LexUriServ.do?uri=OJ:L:1999: 166:0001:00 05:FR:PDF].

Richir M., 1993. Affectivité, Encyclopédia Universalis, Vol. 1, Paris, p. 347-353.

Scannell L., Gifford R., 2010. Defining place attachment : A tripartite organizing framework, Journal of Environmental Psychology, Vol. 30, n 1, p. 1-10.
Simmel G., [1903] 2007. Les grandes villes et la vie de l'esprit, Paris, L'Herne, 59 p.

Thrift N., 2004. Intensities of feeling : Towards a spatial politics of affect, Geografiska Annaler, Vol. 86 B, n 1, p. 57-78.

Tuan Y.-F., [1974] 1990. Topophilia : a study of environmental perception, attitudes and values, New York, Columbia University Press, $260 \mathrm{p}$.

Winnicott D. W., [1971] 1975. Jeu et réalité : l'espace potentiel, Paris, Gallimard, 218 p. 